\title{
A polymeric cell stretching device for real-time imaging with optical microscopy
}

\author{
Yuli Huang • Nam-Trung Nguyen
}

Received: date / Accepted: date

\begin{abstract}
This paper reports the design, fabrication and characterization of a cell stretching device based on the side stretching approach. Numerical simulation using finite element method provides a guideline for optimizing the geometry and maximizing the output strain of the stretched membrane. An unique PDMS-based micro fabrication process was developed for obtaining high parallelization, well controlled membrane thickness and an ultra-thin bottom layer that is crucial for the use with confocal microscopes. The stretching experiments are fully automated with both device actuation and image acquisition. A programmable pneumatic control system was built for simultaneous driving of 24 stretching arrays. The actuation signals are synchronized with the image acquisition system to obtain time-lapse recording of cells grown on the stretched membrane. Experimental results verified the characteristics predicted by the simulation. A platform with 15 stretching units was integrated on a standard $24 \mathrm{~mm} \times 50 \mathrm{~mm}$ glass slide. Each unit can achieve a maximum strain of more than $60 \%$. The platform was tested for cell growth under cyclic stretching. The preliminary results show that the device is compatible with all standard microscopes.
\end{abstract}

Keywords Mechanobiology · Polymeric micromachining · Cell stretching · Microfluidics · Real-time imaging

\author{
Y. Huang \\ School of Mechanical and Aerospace Engineering \\ Nanyang Technological University \\ 50 Nanyang Avenue, Singapore 639798, Singapore \\ N. T. Nguyen \\ Queensland Micro and Nanotechnology Centre \\ Griffith University \\ 170 Kessels Road, Brisbane, 4111, Australia Tel.: +61-7-3735 3921 \\ Fax: +61-7-3735 8021 \\ E-mail: nam-trung.nguyen@griffith.edu.au
}




\section{Introduction}

Cells responded to externally applied mechanical stimuli leading to physiological changes. The study of mechanobiology, which was established around the end of the last century, devotes to studying the relationship between the various mechanical stimuli and cell activities, such as metabolism, biosynthesis, proliferation and apoptosis (Ingber 2003). In the last decade, many commercial or custom-made devices were developed for applying physical stimulus to cells with sizes varying from centimeter to micron scale. Although these devices have their own advantages, only a few of them meet all requirements of biological studies such as minimal consumption of expensive cell sample and reagents, accurate and consistent stimulation strength, high experimental throughput and capability of real-time imaging (Moraes et al. 2011)).

The simplest way to induce a mechanical strain on a cell is directly pulling it with a tool such as a micro pipette (Davis et al. 1992, Maniotis et al. 1997).However, this technique is laborious and does not suit for long-term and high-throughput experiments. Current main-stream devices used for cyclic cell stretching are still implemented in macro scale. One of the most common stretching device is the Flexercell system (FlexCell International, PA) (Flexcell 2007). In this device, cells were seeded on a circular elastic membrane, which is clamped at its edge and is inflated or deflated by the applied positive or negative air pressure (Flexcell 2007). Flexcell systems have been extensively used in mechanobiology research (Garvin et al. 2003, Shi et al. 2011). A common approach is using gel construct for cyclic stretching (Vanderploeg et al. 2008) where cells were seeded into liquid fibrin hydrogel conditioned with culture solution. Mechanical pulling using stepper motors or controllable linear stages was the common actuation technique (Lindqvist et al. 2010, Pfister et al. 2004, Mizutani et al. 2007, Nguyen et al. 2012). Stretching can be achieved wirelessly using magnetically actuated microposts (Sniadecki et al. 2008). After the hydrogel solidifies in the molds and takes its shape, the whole system is mounted onto a tension rake for subsequent application of cyclic tension (Huang et al. 2010, Lee et al. 2010, Wang et al. 2010). Another popular approach is to seed cells onto a polyethylene terephthalate (PET) substrate, which is supported by two pivots and bends as a beam under the loading at its ends (Brown et al. 1998; Mawatari et al. 2010; Owan et al. 1997).

Macro scale devices usually consume a large amount of sample cells. Their large footprint makes them incompatible with conventional microscopes. Therefore, macroscale stretching devices gradually lose popularity to the emerging microscale counterparts. For instance, the adaptation of the membrane distension mechanism in a microfluidic device has been used for microscale cell stretchers (Shimizu et al. 2011; Wu et al. 2011; Kamotani et al. 2008; Tan et al. 2008). Shimizu et al. (2011) used eight balloons with cultured cells connected in series along a naturally occurred pressure gradient, such that different stretching amplitudes can be applied simultaneously. Wu et al. (2011) reported a similar approach with twelve chambers connected to four parallel pressure lines which are independently controlled. This device allowed four 
different loading conditions. In contrast to the device of Shimizu et al., the perfusion based method used in the device of Wu et al. (2011) provided sufficient nutrient to the cells in long term experiments.

In the above mentioned designs, the membrane is deformed into a spherical balloon with a non-uniform strain. An improved stretching approach was proposed by Moraes et al. (2011) using a cylindrical post with a flat tip. The post pushes and stretches the membrane. Thus, the central area of the membrane experiences a uniform equibiaxial strain. Moraes et al. (2011) integrated an $9 \times 12$ array of stretching units in single device. By varying the size of the cavity, a set of strain amplitudes can be achieved with the single pressure source. Further modification of this stretching approach replaces the moving post with a stationary post (Simmons et al. 2010). The membrane was stretched by applying vacuum on its rim, keeping it in the focal plane of a microscope throughout a stretching cycle. Thus, this concept allows real-time recording of the cells. The ability of observing the cells in real time under high optical magnification is an important requirement for the study of mechanobiology from colony scale to cellular or sub-cellular scale (Moraes et al. 2011). However, the postsupporting approach suffers under the high friction and requires a lubricant to operate. The common membrane material polydimethylsiloxane (PDMS) reacts with silicon-based lubricant. And most of industrial lubricants degrade the membrane over time. Even for water-based lubricant, the stretching device was only tested for a continuous operation up to 6 hours. Furthermore, a humidified environment is needed for reducing evaporation of the lubricant (Simmons et al. 2010). Another problem is the porosity of PDMS leading to the permeation of lubricant into the cell side contaminating the fragile microenvironment. Most importantly, the large working distance is the bottleneck of existing macroscale devices working with a microscope. A short working distance required for real-time imaging can only be achieved with a microdevice.

A recent stretching device originally designed for reconstructing the organlevel lung function (Huh et al. 2010) provides an alternative for in-focus cell stretching. Vacuum was applied to two side chambers where the ends of membrane are attached, Fig. 1. Since the membrane is attached to the centre of the chamber wall, the actuation does not cause any vertical motion. The membrane is always stretched horizontally in the focal plane of the microscope. The simplicity of this approach makes it suitable for a high level of parallelization and miniaturization. The closed-chamber design can effectively isolate the microenvironment from external contamination. The present paper focuses on the mechanical design and optimization of this stretching approach. As a further development, an array of stretching units is integrated into a single device. A fabrication process with the possibility of scaling up was developed for the device. A complete control system for synchronized actuation and image acquisition was also developed. The device was characterized and tested with cell cultures. Finally, experimental data are discussed and compared with numerically simulated data. 


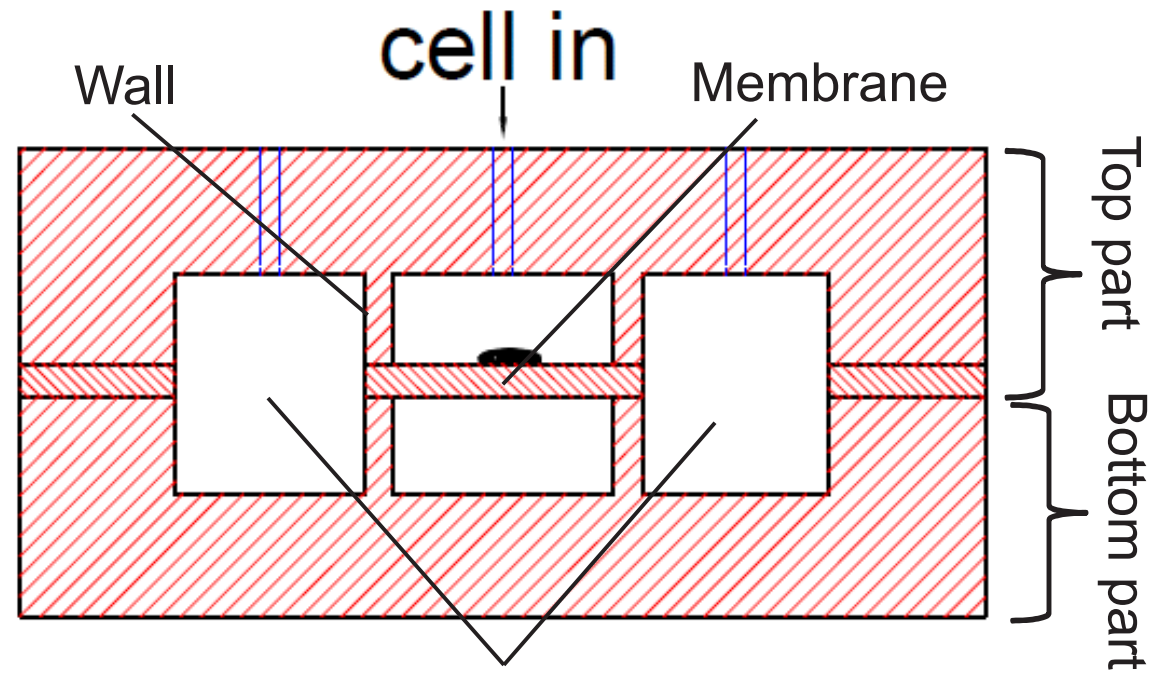

(a) Vacuum chambers
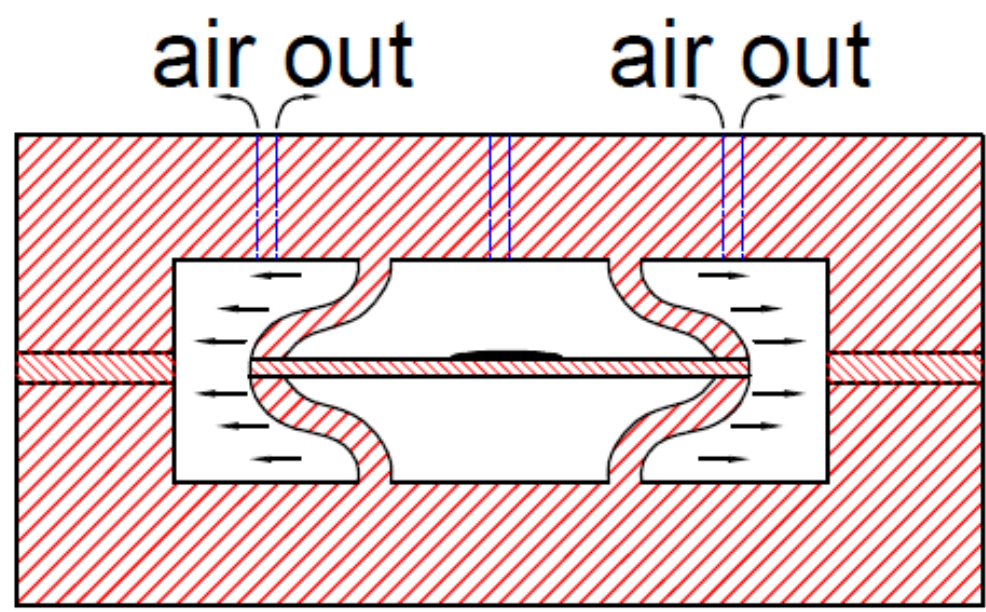

(b)

Fig. 1 Illustration of the working principle of the side stretching approach: (a) Relaxed; (b) Stretched.

\section{Design and numerical simulation}

In the conceptual design phase, finite element analysis (FEA) was used to predict the resultant strain distribution on the membrane. The numerical model provides a design guideline for the stretching unit in the actual fabricated device. Reasonable simplifications were made to save the computational expense. First, the stretched membrane is considered as a plane. Second, the 


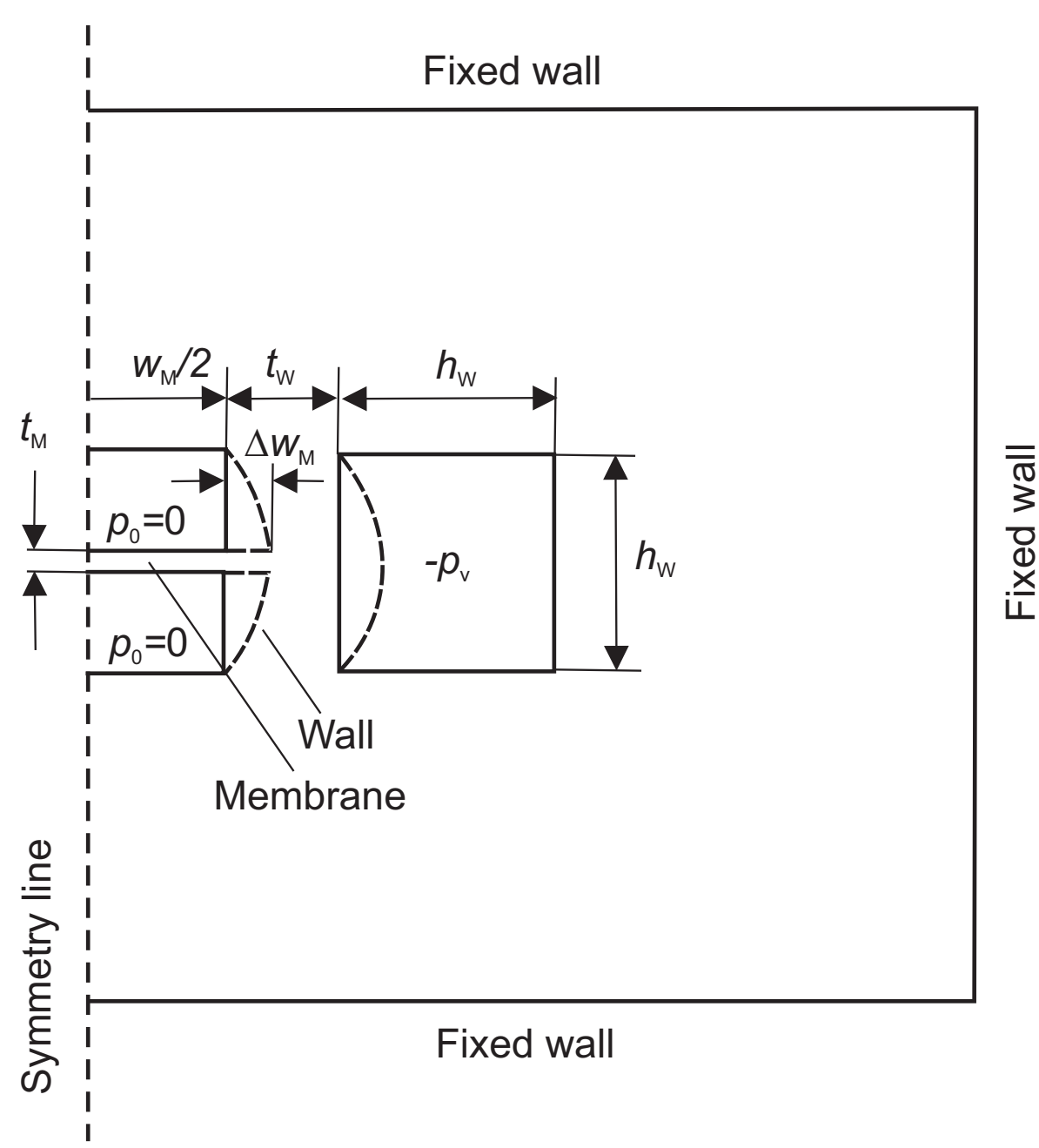

Fig. 2 The cross-section of the 2D FEA model with the parameters of interest.

deformation occurs only in the membrane and neighboring region of the vacuum chambers. Third, the mechanism is plane-symmetric about the central vertical plane. Fourth, the material properties of PDMS are taken as linearly elastic and time independent. With these assumptions, a two-dimensional (2D) plane strain model (element type PLANE82) was used in the commercial software ANSYS (ANSYS Inc, USA) for the static structural analysis of the right half cross-section of the device. Figure 2 shows the model with the key parameters used for the optimization. The reference design has the following parameters: membrane thickness $t_{\mathrm{M}}=8 \mu \mathrm{m}$, membrane width $w_{\mathrm{M}}=250 \mu \mathrm{m}$, wall thickness $t_{\mathrm{W}}=100 \mu \mathrm{m}$ and wall height $h_{\mathrm{W}}=200 \mu \mathrm{m}$. For a better accuracy, the membrane, the wall and the neighboring outer region was meshed with $2 \mu \mathrm{m}, 4 \mu \mathrm{m}$ and $8 \mu \mathrm{m}$ element size, respectively. The material properties 


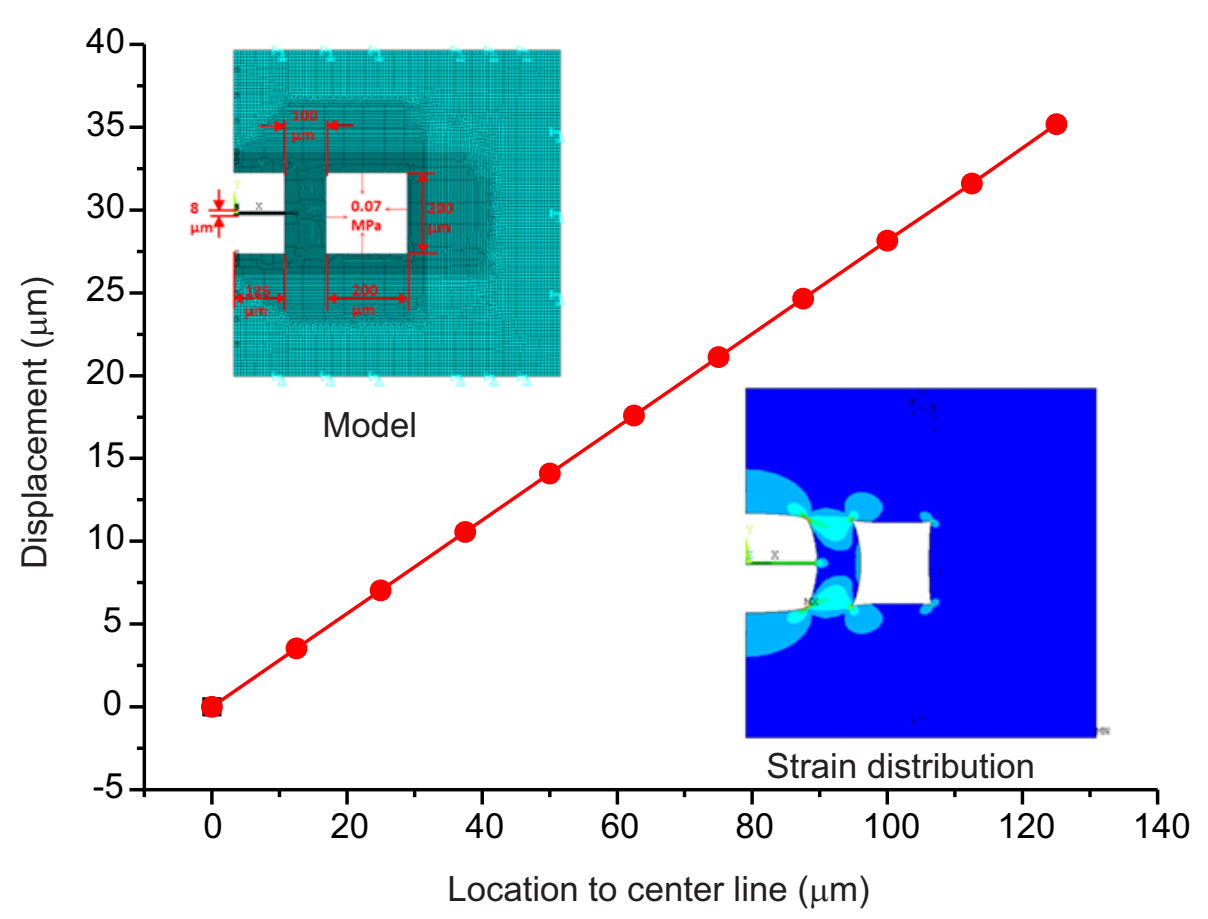

Fig. 3 Simulated displacement of the membrane versus the location from the center line of the membrane or the symmetry line of the model $\left(t_{\mathrm{M}}=8 \mu \mathrm{m}, w_{\mathrm{M}}=250 \mu \mathrm{m}, t_{\mathrm{W}}=100 \mu \mathrm{m}\right.$, $\left.h_{\mathrm{W}}=200 \mu \mathrm{m}, p_{\mathrm{V}}=70 \mathrm{kPa}\right)$.

of PDMS were taken as linearly elastic with an Young's Modulus of 0.5 MPa and a Poisson's ratio of 0.5 . The boundary condition is applied such that the region of interest (ROI) is fully confined in fixed walls, meaning all degrees of freedom (DOF) at the outer edges are set to zero. This assumption is valid because the outer wall of the actual device in millimeters) is three orders of magnitude larger than the features of the device (in micrometers). A negative pressure is applied on all sides of the vacuum chamber wall to represent the actuating vacuum $-p_{\mathrm{V}}$. The reference pressure $p_{0}$ around the membrane is set to $p_{0}=0$. Figure 3 shows the displacement of the membrane versus the location from its centreline for the basic design under a vacuum strength of $p_{\mathrm{V}}=70 \mathrm{kPa}$. The result indicates a linear relationship and thus an uniform strain along the transversal direction of the membrane surface.

Parametric optimization was carried out by varying one geometry parameter while keeping all other parameters at the reference values. Figure 4 shows the relationship between the lateral displacement of the membrane as a function of varying wall height, wall thickness, membrane thickness, and vacuum strength, respectively. The results indicate that the key parameters are the wall thickness and the membrane thickness. The membrane width produces a marginal effect on the overall displacement and strain. The structure defor- 

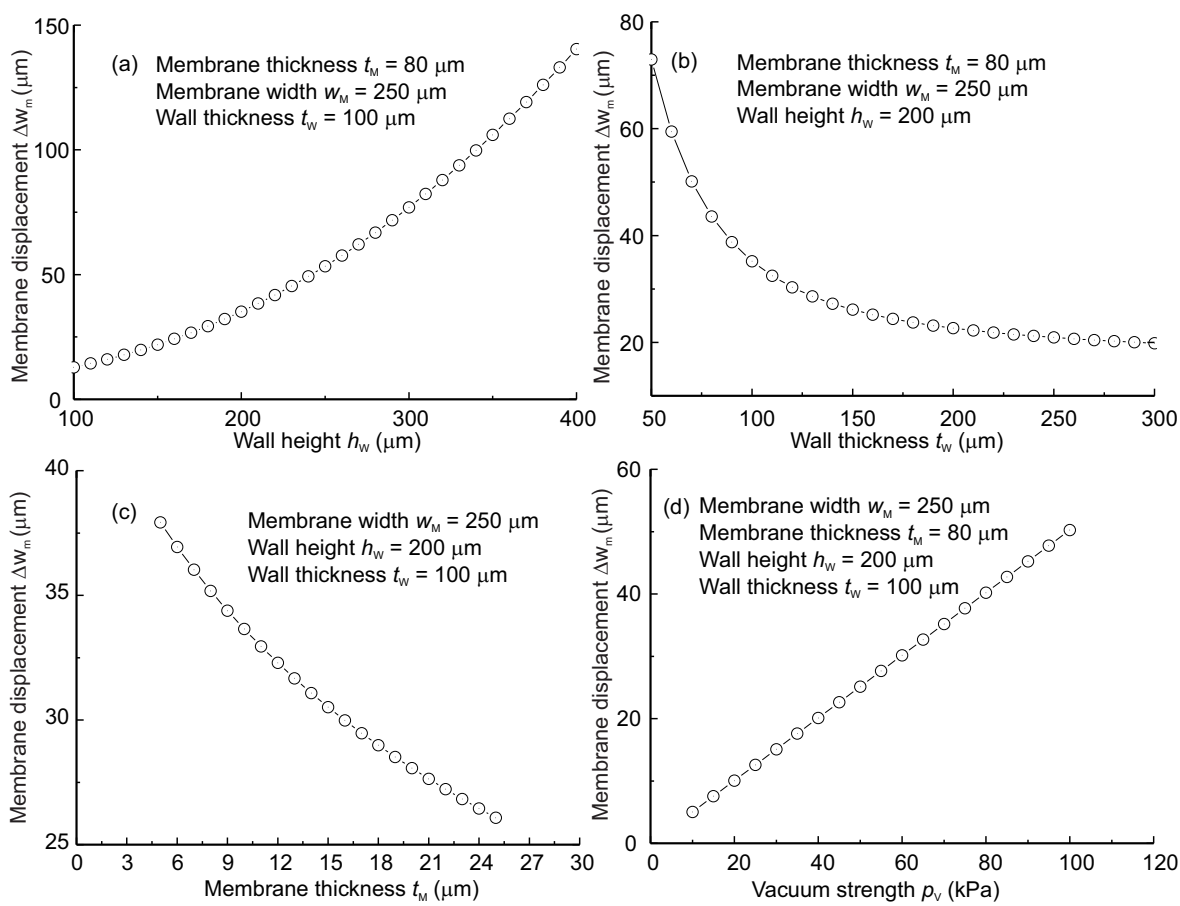

Fig. 4 Parametric optimization for the lateral displacement of the membrane: (a) Wall height $h_{\mathrm{W}}$; (b) Wall thickness $t_{\mathrm{W}} ;$ (c) Membrane thickness $t_{M} ;$ (d) Vacuum strength $p_{\mathrm{v}}$

mation is mainly affected by the deflection of the wall. The results indicate that both the wall height [Fig. 4(a)] and wall thickness [Fig. 4(b)] have a large impact on the resultant strain. While the lateral displacement of the membrane grows almost exponentially with the wall height, a significant change can only be expected with a wall thickness ranging from $50 \mu \mathrm{m}$ to $120 \mu \mathrm{m}$. The lateral displacement of the membrane decreases almost linearly with the increasing membrane thickness, Fig. 4(c). Figure 4(d) shows that the lateral displacement $\Delta w_{\mathrm{M}}$ (Fig. 2) of the membrane increases linearly with the applied vacuum strength. This linear relationship makes real-time control of the strain output straightforward. The above parametric study suggests that the wall height of the actual stretching unit should be maximized, while both the wall thickness and the membrane thickness should be reduced. However, due to the limitations of the fabrication technology and the conflicting requirements of other design objectives such as a short optical working distance, the wall height can only vary from $100 \mu \mathrm{m}$ to $400 \mu \mathrm{m}$. The wall thickness varies between $50 \mu \mathrm{m}$ and $100 \mu \mathrm{m}$. Due to the given recipe of a reproducible thin PDMS membrane as reported in the next section, the membrane thickness is fixed at $8 \mu \mathrm{m}$. 


\section{Device fabrication}

The fabrication process of the device consists of three major steps: preparation, PDMS molding and final assembly. In the first step, a glass slide was precoated with octafluorocyclobutane $\left(\mathrm{C}_{4} \mathrm{~F}_{8}\right)$ using a deep reactive-ion etching system (STS, 1 minute), where only the coating step was activated, Fig. 5(a). A coverslip (Fisher Scientific, $24 \mathrm{~mm} \times 50 \mathrm{~mm} \times 170 \mu \mathrm{m}$ ) was attached to the coated glass slide by tapping its two long edges onto the glass slide using a Kapton $^{\text {TM }}$ tape (3M, USA), Fig. 5(b). The thin and fragile glass coverslip is needed for optical access with a microscope. The thicker glass slide provides structural support for the thin coverslip and the PDMS device throughout the fabrication process. Since the bottom part and the top part of the PDMS device are identical, both were fabricated with the same master mold. The master mold was fabricated using standard SU-8 photolithography process. First, a clean silicon wafer was baked at $150^{\circ} \mathrm{C}$ for 20 minutes to remove possible water residue. SU-8 was then uniformly dispensed onto the silicon wafer, and spin coated (Delta 80BM, SUSS MicroTec) at a speed of $1350 \mathrm{rpm}$ for 60 seconds to achieve a thickness of $200 \mu \mathrm{m}$, Fig. 5(c). The thickness of the SU-8 layer determines the height of the wall. The wafer was then prebaked at $95^{\circ} \mathrm{C}$ for 5 minutes. Due to the large thickness of SU- 8 used, the temperature was slowly ramped up from room temperature to avoid cracks. Subsequently, the pattern of the device was transferred to the SU-8 layer through UV exposure, Fig. 5(d). A photolithography mask printed on a plastic transparency with a resolution of 9600 dpi (Infinite Graphics, Singapore) was first mounted on the mask aligner (MA6, SUSS MicroTec), UV radiation (350 nm-400 nm) was then activated. The uncovered areas of the SU- 8 was exposed to the radiation with an intensity of $9.3 \mathrm{~mW} / \mathrm{cm}^{2}$ for 175 seconds. Post-baking of the wafer was subsequently carried out at $95^{\circ} \mathrm{C}$ for 30 minutes facilitating further cross-linking of the exposed SU-8. The wafer was then immersed in the SU-8 developer solution (MicroChem) until the non-exposed area was fully washed away, Fig. 5(e). The development process usually took about 15 minutes for a thickness of $200 \mu \mathrm{m}$. The SU-8 mold was also coated with $\mathrm{C}_{4} \mathrm{~F}_{8}$ in the same way as mentioned above for the glass slide to ease the later release of PDMS, Fig. 5(f).

In step two, the bottom and the top PDMS layers were fabricated. The bottom PDMS layer with the thin glass coverslip was casted by pressing the master mold to the supported coverslip, Fig. 6(a). A $40 \mathrm{kPa}$ static pressure from a steel block was then loaded on the coverslip. This layer was cured at $80^{\circ} \mathrm{C}$ for 1 hour. After releasing the mold, a PDMS structure with $200 \mu \mathrm{m}$ thickness was left on the glass coverslip, Fig. 6(b). The top PDMS layer was fabricated by transferring the thin membrane to the molded PDMS part with access holes. The PDMS mixture was first poured on the master mold, than vacuumed to remove air bubbles. This layer was cured at room temperature for 48 hours, so that pattern distortion due to thermal expansion can be avoided, Fig. 6(c). Access holes were opened on the top PDMS layer using a manual puncher (Harris Uni-Core 0.5 to $1 \mathrm{~mm}$, Sigma-Aldrich). The thin PDMS membrane was spin coated on a silicone wafer at $4000 \mathrm{rpm}$ for 3 minutes. 


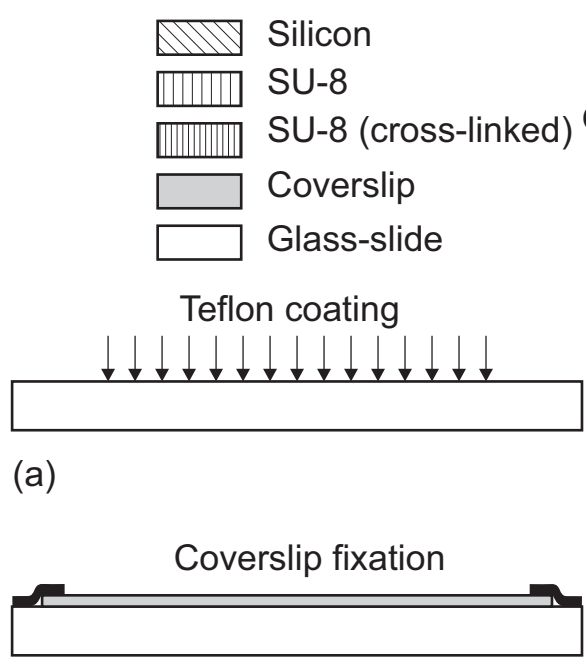

(b)

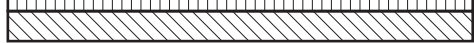

(c) UV exposure

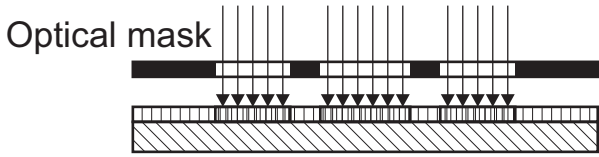

(d)

SU-8 development

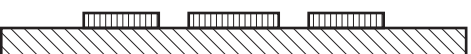

(e)

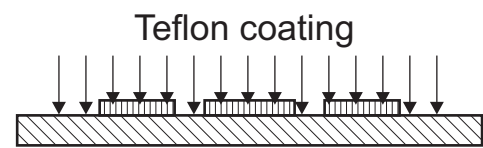

(f)

Fig. 5 Preparation step: (a,b) Preparation of the thin glass coverslip; (c-f) Fabrication of the SU-8 master mold.

This recipe gives a final reproducible thickness of $8 \mu \mathrm{m}$ using our equipment. The coated PDMS membrane was then bonded to the top layer using oxygen plasma (NT-2, BSET EQ, $120 \mathrm{~W}, 30 \mathrm{~s}$ ), Fig. 6(c). The stack was cured together at room temperature for 10 minutes. Upon completion, the portions of the membrane that blocked the access-holes were manually removed using tweezers with an ultra-fine tip (Cole-Parmer), firstly to make the space bellow the mebrane accessible and secondly to connect the top and bottom halves of vacuum channels. This manual process could be replaced by an etching process reported next.

In step three, the top and bottom layers are aligned and bonded together to form the final device. The challenge here is the precise alignment of the two layers and the speed of this process due to the limited time available for the surface activated by oxygen plasma. The activation of the PDMS surface only lasts for about 5 minutes. Alignment and bonding should occur in this time frame. Only one touch between the two layers will cause permanent bonding. Thus, accurate alignment is very crucial for the whole assembly process. We developed an alignment system for this purpose. The alignment system is able to precisely position an object with four degrees of freedom (DOF), namely $\mathrm{X}, \mathrm{Y}, \mathrm{Z}$-translational and Z-rotational. Furthermore, a flip-up and screw tightening mechanism ensures quick mounting and dismounting of the PDMS parts needed for fast bonding. The device was mounted on the motorized stage of an inverted microscope with $4 \times$ magnification, Fig. 7 (a). Prior to the plasma treatment, the bottom PDMS layer with the coverslip and glass-slide attached to it was locked on the microscope stage. The top layer was temporarily at- 


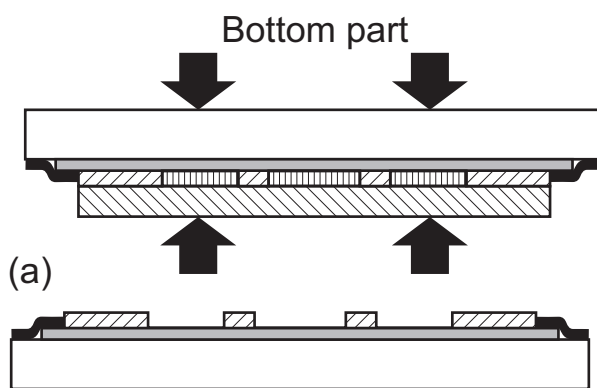

(b)
Top part

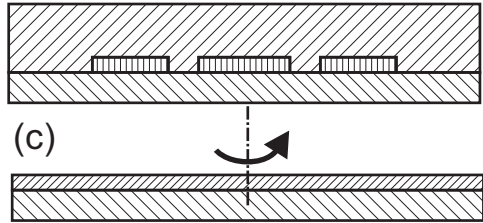

(d)

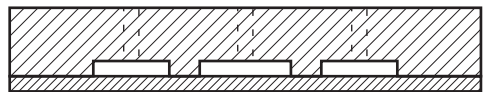

(e)
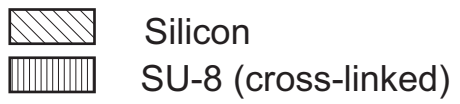

Coverslip Glass-slide WIIA PDMS substrate

Fig. 6 PDMS molding step: (a,b) Fabrication of the bottom layer; (c-e) Fabrication of the top layer with the transferred membrane.

tached to a glass-slide on its back, and locked on the sample holder of the alignment device. After the focus was found and coarse alignment was made [Fig. 7(b)], both pieces were unlocked by the quick-release mechanisms and brought for simultaneous plasma treatment. Immediately afterwards, they were locked back into the alignment position again. Fine alignment was performed and these two pieces were eventually brought into contact, Fig. 7(c). The whole assembly was then transferred into the oven $\left(50^{\circ} \mathrm{C}, 10 \mathrm{~min}\right)$ to stabilize the bond.

If the membrane area in the vacuum chambers [Fig. 8(a)] are not removed mechanically as described above, it can be etched away in a subsequent step. A PDMS etchant consisting of a mixture of 3:1 volume ratio of tetra-n-butylammonium fluoride (TBAF) and dimethylformamide (DMF)) was pumped through the vacuum channels and removes the membrane, Fig. 8(b). The disadvantage of this method is that the actuating wall is also etched and thinned. Etching time and flow rate of the etchant should be controlled precisely to obtain the desirable wall thickness. In the end, the glass-slide that was temporarily used as structural support for the top part was detached from the stack. The Kapton ${ }^{\mathrm{TM}}$ tapes were removed, and the coverslip was carefully freed from the supporting glass slide. Sliding was used instead of peeling to avoid cracking of the fragile coverslip. Finally, the bottom of coverslip was thoroughly washed with ethanol to obtain good optical clarity, Fig. 8(c).

An array of 15 stretching units was integrated on one chip with the size of $24 \mathrm{~mm} \times 50 \mathrm{~mm}$ of a standard coverslip to increase the experimental throughput, Fig. 9(a). Every three units in a column share one common vacuum port, creating a statistically meaningful triplicate condition for the later experiments. In most cases where the strain magnitude is to be varied, a configuration with strain gradient was designed. The membrane widths were ad- 


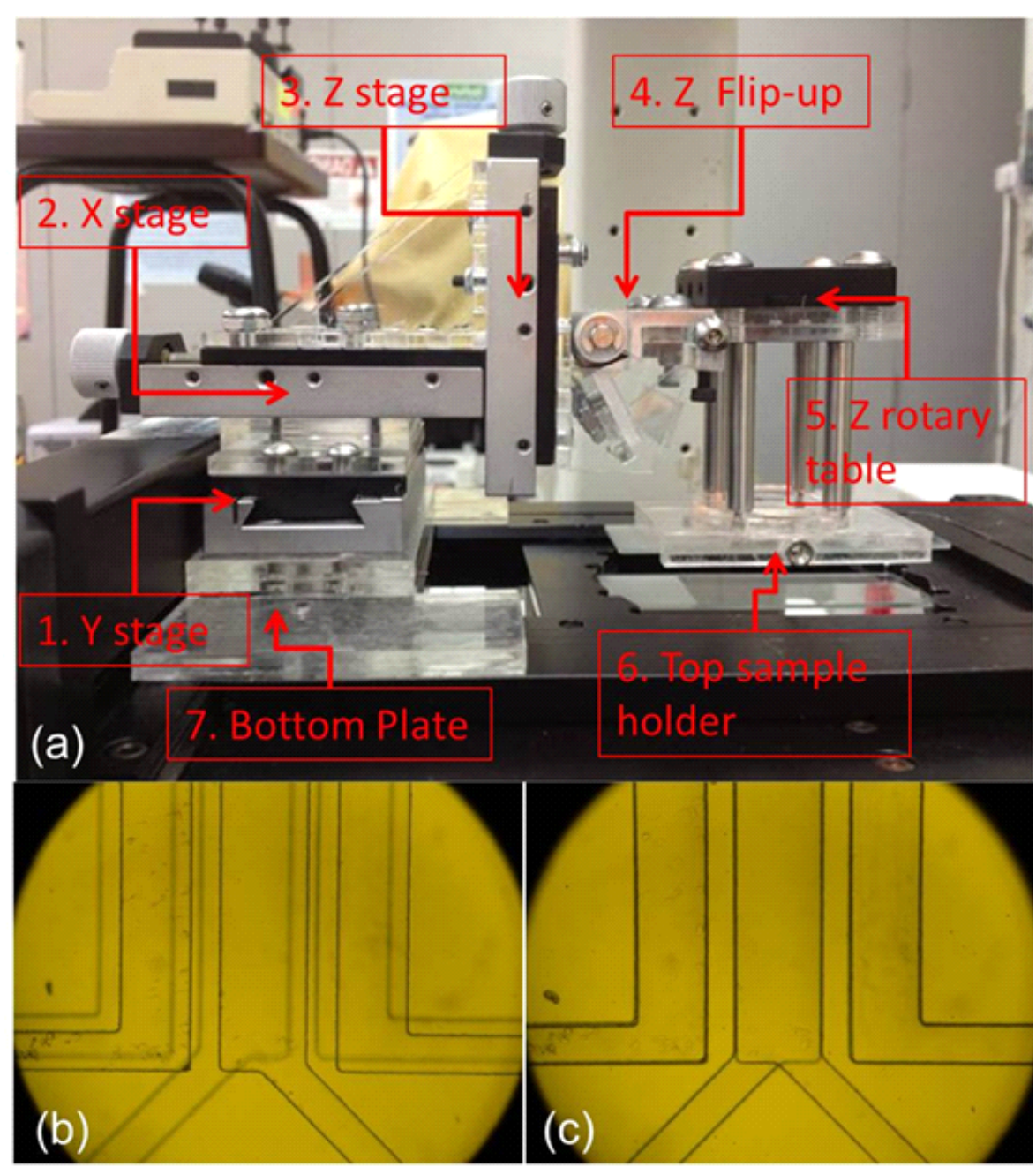

Fig. 7 Alignment of the top and bottom layers: (a) The setup of the 4 DOF alignment and bonding equipment; (b) Misalignment; (c) Alignment seen through a microscope with $4 \times$ magnification.

justed according to the relationship: $\varepsilon=2 \Delta w_{\mathrm{M}} / w_{\mathrm{M}}$, where $\varepsilon$ is the required maximum strain, $\Delta w_{\mathrm{M}}$ is the maximum lateral displacement, and $w_{\mathrm{M}}$ is the channel width as shown in Fig. 2. In our case, varying the membrane width of the stretching units results in five linearly incremental strains that can be simultaneously applied in a single experiment, Fig. 9(b). 
(a)

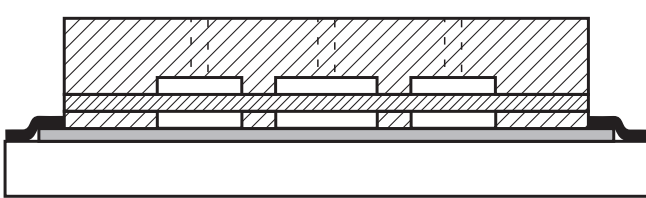

\begin{tabular}{|ll}
\hline & Coverslip \\
\hline & Glass-slide \\
VIIIA & PDMS membrane \\
DIIA & PDMS substrate
\end{tabular}

(b)

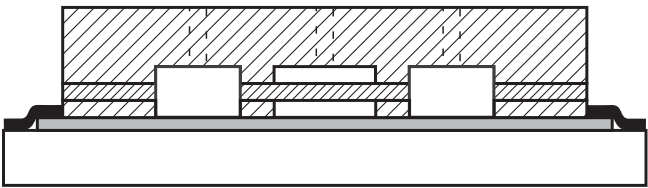

(c)

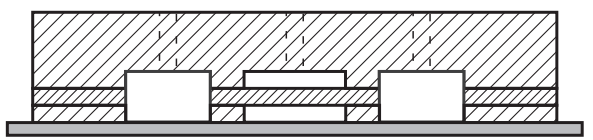

Fig. 8 Alignment and assembly step: (a) Assembly stack after bonding; (b) Assembly stack after removing the membrane in the vacuum chambers; (c) The final stretching unit.

\section{Control system for synchronized pneumatic actuation and image acquisition}

Other than the strain magnitude, the strain frequency is another important parameter to be investigated for cyclic stretching experiments. Our control system was designed with the capability of providing separate and variable frequency to each column of stretching units. A multi-valve pneumatic control system was built. The system consists of a vacuum pump (Dryfast 2034, Welch, USA), a vacuum controller (LabAid 1640, Welch, USA), an array of $8 \times 3$ solenoid valves (S10MM-31-12-3, Pneumadyne, USA). The control program was written in Labview. Through a Labview user interface (UI), user inputs are passed to a current amplifying circuit. This current amplifying circuit sent 24 individual signals simultaneously to the respective solenoid valves and turn them on and off accordingly. Thus, the stretching units in different columns can be concurrently actuated with different frequencies. Figure 10 shows the schematic of our control system.

The consistency of the state of the membrane is important for multi-point time-lapse image recording of long-term cell experiments, which usually lasts for several hours or more. The series of images of an area loaded with cells should always be taken when it is in the relaxed state, so that its morphology at different time instances can be meaningfully compared. An interruption system was added to the actuation system to synchronize actuation with image acquisition. The interruption system works as follows. A TTL interrupt signal is sent out before each image acquisition time instance. The time difference between the interrupt and acquisition is called the time delay. It is usually set at $50 \mathrm{~ms}$ from the microscope control software (Metamorph, Molecular Devices, USA) to give the pneumatic system enough time to get ready for each image acquisition. The interrupt signal is modulated by a 555 timer circuit, 


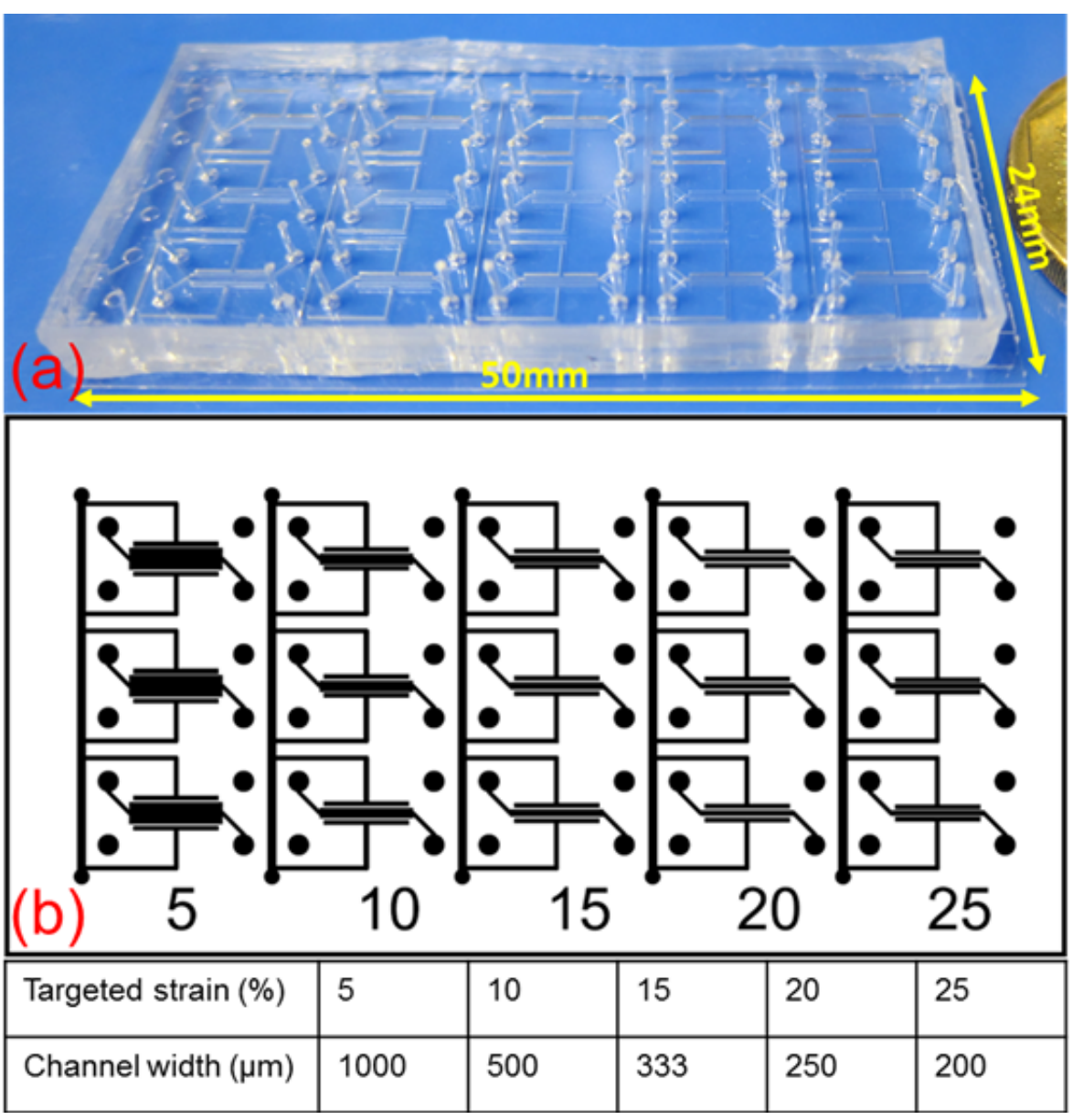

Fig. 9 The cell stretching device with an array of $3 \times 5$ stretching units: (a) The assembled device. (b) The device layout and the mapping between targeted strain and the membrane width width.

which inverts the pulse polarity and extends the duration to a user specified one. The pulse duration should be set long enough to cover one multi-point image acquisition. The setting of the pulse duration is done via a trim-pot on the circuit. An AND logic was applied to the modulated interrupt signal and the actuation pulse train, which causes an interruption in the pulse train to the pneumatic control system during the time of image acquisition. Therefore, the valves were always deactivated while image acquisition is in progress. Thus, image recording only occurs when the cells are in the relaxed state.

The range of applicable frequency is limited by the electrical response of the solenoid and the performance of the vacuum pump. The electrical response time of the valve was given by factory specification as $10 \mathrm{~ms}$. Following estimation was performed to understand the limitation of the vacuum 


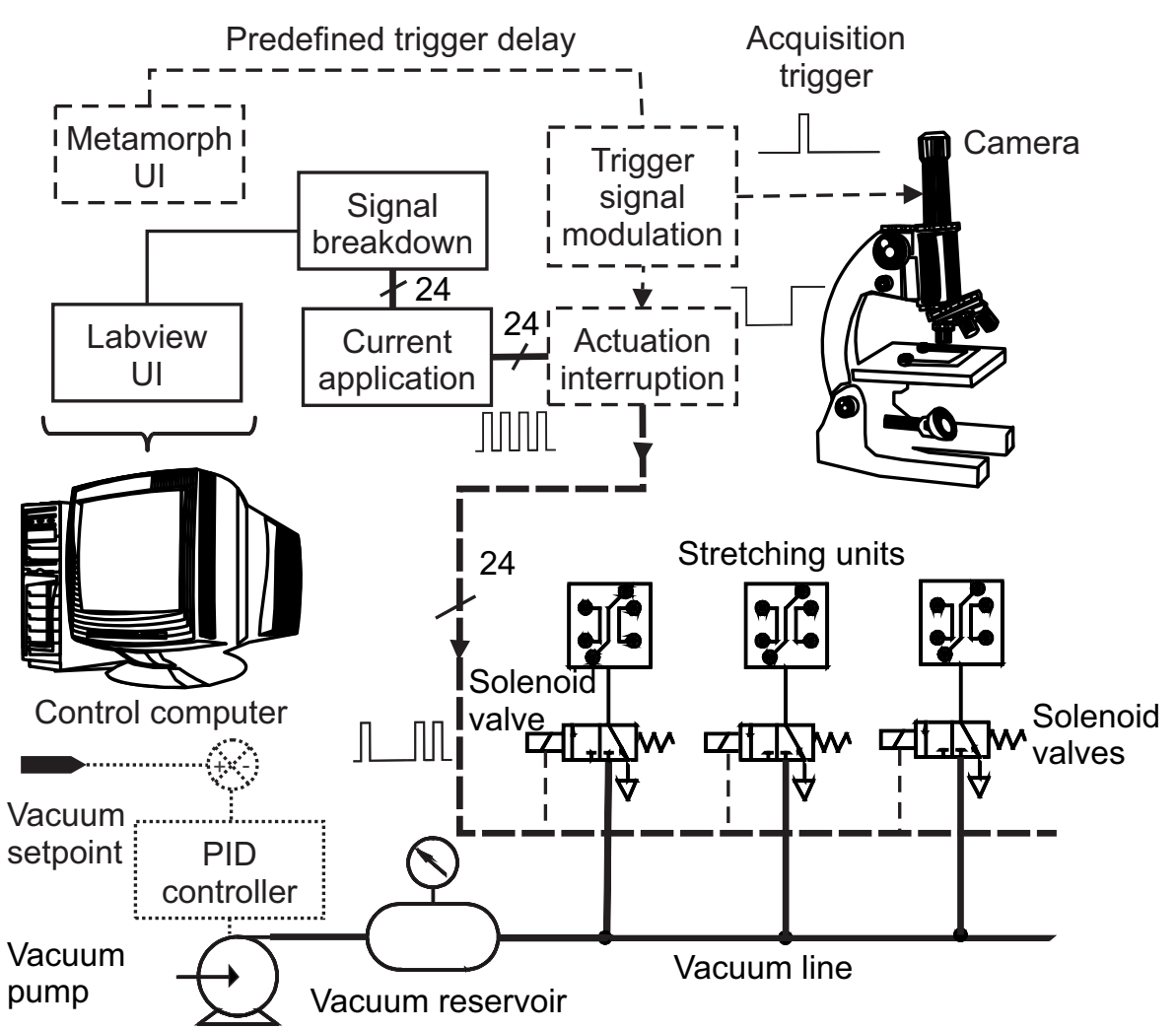

Fig. 10 The schematics of control system, with vacuum control subsystem (dotted lines), pneumatic subsystems (thick solid line), microscope subsystem (dashed lines), valve actuation subsystem before interruption (solid lines) and after interruption (thick dashed lines).

pump. Figure 10 shows that cyclic stretching of each unit was controlled by the 3-way 2-position normally-closed solenoid valve. In the stretching state, vacuum occupies each stretching unit and their respective connecting tubing. In the relaxed state, the vacuum was cut off by the valve. The space was then filled by atmospheric pressure. The requirement for the vacuum pump was defined as being capable of equalizing the pressure in each unit within the stretching half-cycle. In other words, the pump has to remove the amount of air that has filled the stretching unit as well as its respective tubing $V_{0}=n\left(V_{\text {tubing1 }}+V_{\text {chamber }}+V_{\text {tubing2 }}\right)$ with $n$ the number of stretching units. For $n=24$ units running in parallel, the volume of our pneumatic system is estimated to be $V_{0}=1.05 \times 10^{-5} \mathrm{~m}^{3}$ within a half-period of each cycle.

A few assumptions were made to analyze the response of the vacuum source. First, as the rigid polytetrafluoroethylene (PTFE) tubing was used, the volume of each unit and its connecting tubing was considered fixed. Second, as the operating condition is under room temperature and low pressure, air can be modeled as an ideal gas. Third, the pump performance curve was 
linearized according to the manufacturer's datasheet as:

$$
Q_{\mathrm{dis}}=k p
$$

where $Q_{\text {dis }}$ is the volumetric rate of air discharged into atmosphere, $k$ is the proportional factor representing the pump performance and $p$ is the absolute pressure in the stretching unit and the tubings. The discharge rate is:

$$
Q_{\mathrm{dis}}=\frac{\mathrm{d}}{\mathrm{d} t} V_{\mathrm{dis}}=-\frac{\mathrm{d}}{\mathrm{d} t} p \frac{V_{0}}{p_{\mathrm{atm}}}
$$

where $p_{\text {atm }}$ is the atmospheric pressure. Combined with the pump performance function, the first order relationship is established for the absolute pressure as:

$$
-\frac{\mathrm{d}}{\mathrm{d} t} p \frac{V_{0}}{p_{\text {atm }}}=k p
$$

Solving the above equation with the initial condition $p=p_{\text {atm }}$ results in the time response of the pneumatic system:

$$
p=p_{\text {atm }} \exp \left(-\frac{k p_{\text {atm }}}{V_{0}} t\right)
$$

Figure 11 shows the response of the absolute pressure under different loads of 24,8 and 1 valves based on the pump performance used in our experiments $\left(k \approx 35 \times 10^{-10} \mathrm{~m}^{3} / \mathrm{sec} \cdot \mathrm{kPa}\right)$. If the pump was used for simultaneous actuation of 24 valves, the time constant is approximately $30 \mathrm{~ms}$, meaning the actuation frequency should be capped at $16.7 \mathrm{~Hz}$. In an experiment that only requires parallelization of less than 8 valves, the driving frequency can be set as high as $50 \mathrm{~Hz}$. Compared to the $10 \mathrm{~ms}$ or $100 \mathrm{~Hz}$ set by the electrical response of the solenoid valve, the pump performance is still the limiting factor for the actuation frequency of the stretching device.

\section{Results and discussion}

\subsection{Strain characterization}

As a proof of concept, fluorescent micro-beads (Thermo Scientific, $1 \mu \mathrm{m}$, EX/EM 542/612 nm, diluted to $0.25 \%$ solid volume) were suspended in water and introduced into the membrane channel (wall height $h_{\mathrm{W}}=400 \mu \mathrm{m}$, wall thickness $t_{\mathrm{W}}=100 \mu \mathrm{m}$, membrane width $\left.w_{\mathrm{B}}=200 \mu \mathrm{m}\right)$. The membrane was cyclically stretched. Two images were taken at the relaxed and the stretched state, respectively. As the membrane was stretched, it exerted a shear stress to the fluid setting the neighboring fluid layer to motion. Particle image velocimetry (PIV) algorithm (OpenPIV) was applied to both pictures to analyze the motion of the micro-beads that also represents the deformation of the membrane, Fig. 12(a). The resultant plot of vectors showed that the motion of beads was a combined effect of fluid flow along the channel direction and the 


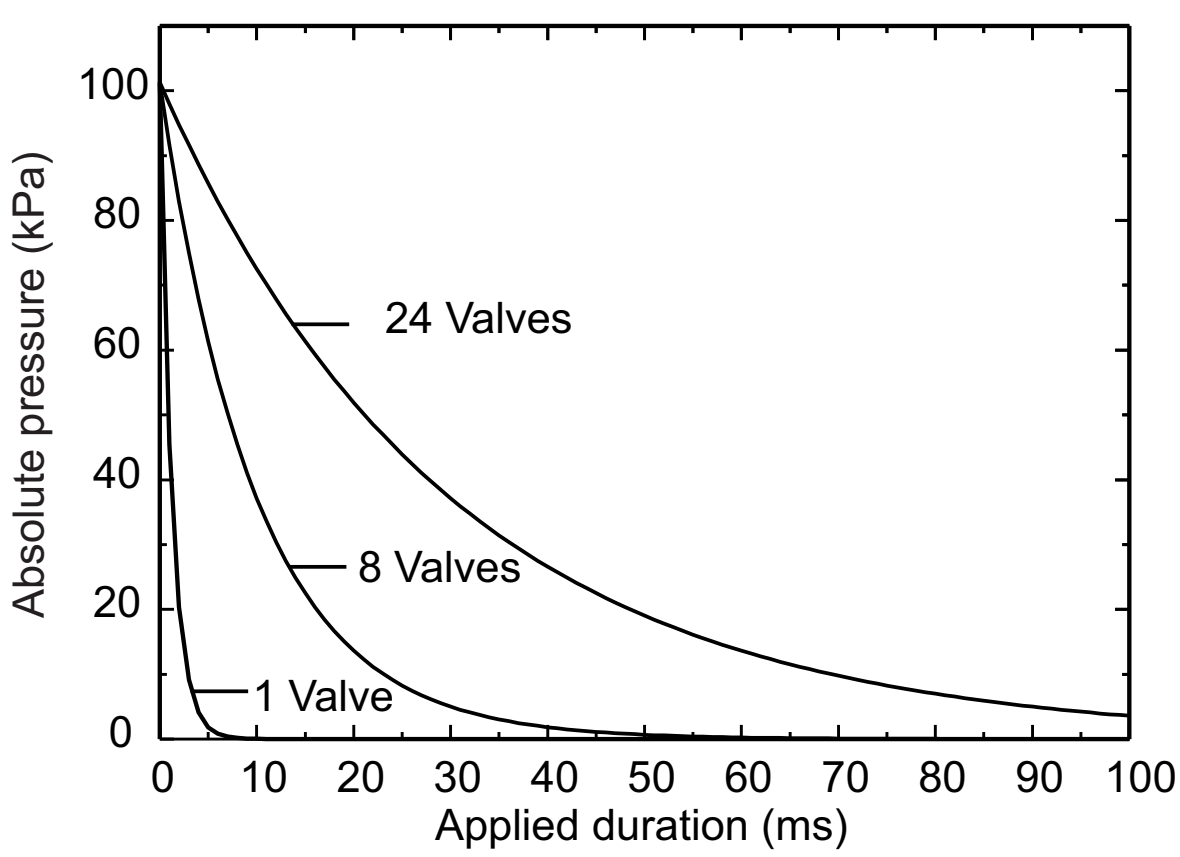

Fig. 11 The pneumatic response with different numbers of stretching units.

bi-directional stretching transverse to the channel. Two important observations can be made here. First, the vertical components of all vectors from the same row were almost equal and slightly smaller near the edges suggesting a laminar flow in the channel above the membrane. Second, the horizontal components of displacement vectors were identical in each row. Within each row, the displacement vectors are distributed in an identical way and increase linearly from the center to the edge of the the membrane. This observation agrees well with the numerical simulation.

To immobilize the particles on the membrane for a more accurate deformation analysis, the beads were transferred onto the membrane via oxygenplasma assisted contact printing. Sequential image of the stretched membrane were taken at discrete vacuum levels ranging from the atmospheric pressure to the maximum of vacuum pump output with a constant increment of 13 $\mathrm{kPa}$. Figure 12(b) shows the displacement analysis of the stretched membrane using the same PIV software mentioned above. All displacement vectors now are aligned in the transverse direction relative to the channel. This result indicates that pure uniaxial stretching condition was obtained with our device. The general trend of a linearly increasing displacement from the center to the edge of the membrane is also confirmed. Furthermore, the area inside the green rectangle shown in Fig. 12(b) was taken to form a kymograph, the graphical representation of spatial position over vacuum strength. On the kymograph, a bead forms a straight line. Thus, the linear relationship between the strain and vacuum strength was also confirmed. More importantly, as all 

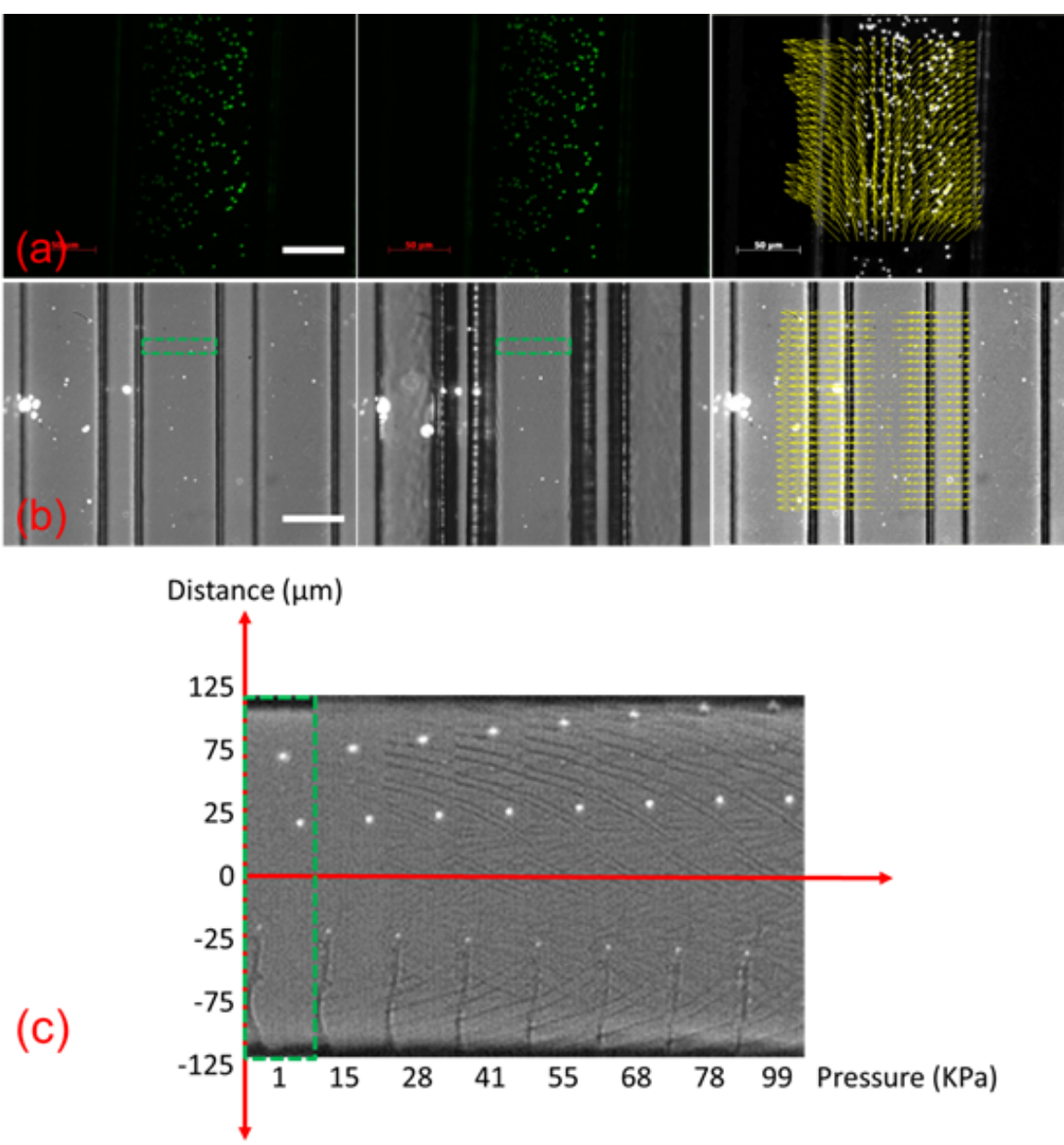

Fig. 12 Characterization of the stretched membrane: (a) The motion analysis of fluorescent particles in a microchannel above the stretched membrane; (b) Displacement analysis of fluorescent particles embedded in the stretched membrane (the scale bars in (a) and (b) are $200 \mu \mathrm{m})$; (c) The graphical representation of spatial position over vacuum strength. The image section framed by the green rectangle in (b) is rotated by $90^{\circ}$ and placed according to the corresponding vacuum strength, forming a kymograph.

the beads remained equally visible and clear throughout the stretching process, the characterization experiment proved that there is no noticeable out-of-focus movement. The stretched membrane was kept entirely in the focal plane of the microscope.

Quantitative analysis of the strain was done by performing measurements (ImageJ, National Institute of Health, USA) on the same series of recorded images. First, the recorded images were stacked in an ascending order of applied vacuum strength. In the first image (relaxed state), the centreline of the membrane was determined by averaging the two edges. Subsequently, 20 beads 
Table 1 Summary of line fitting results for displacement of the membrane.

\begin{tabular}{rrrr} 
Vacuum strength $p_{\mathrm{v}}(\mathrm{kPa})$ & Gradient & y-intercept $(\mu \mathrm{m})$ & $R^{2}$ \\
\hline 18.7 & 0.117 & -0.309 & 0.970 \\
32.0 & 0.215 & 0.438 & 0.979 \\
45.3 & 0.341 & -0.860 & 0.988 \\
58.7 & 0.472 & -1.387 & 0.995 \\
72.0 & 0.551 & -1.389 & 0.995 \\
85.3 & 0.622 & -0.692 & 0.994 \\
98.7 & 0.673 & 0.368 & 0.995 \\
\hline
\end{tabular}

were identified and their respective locations in each image were tracked. The locations of beads in the first image relative to the location of the centreline were taken as their original locations. Similarly, the distance moved by each bead from its original location to the new position in the $i$ th image was recorded as its displacement $\delta_{i}=x_{i}-x_{0}$. The corresponding strain can be determined as $\varepsilon_{i}=\delta_{i} / x_{0}$. The displacement and strain are plotted as versus the location in Figures 12(a) and (b), respectively. Each data set represents the displacement and strain at a given vacuum strength. Linear fittings were carried out for each data set to depict the trend of the data.

Table 1 shows that linear fitting of the displacement produces coefficients of determination $\left(R^{2}\right)$ greater than 0.97 at all levels confirming the linear relationship predicted by the numerical simulation, Fig. 3. As the displacement magnitude increases with the vacuum strength, the $R^{2}$ value approaches 1 indicating that a larger magnitude will usually produce a smaller relative error. Additionally, all fitted lines in the displacement data converge at one point. The $y$-intercepts are within the range of $\pm 1.5 \mu \mathrm{m}$. The convergence suggests that stretching occurs along the geometric centerline of the long rectangular membrane. The assumption of plane symmetry used in the numerical simulation is also validated here.

Comparing the strain data on Fig. 13(b), a constant increment with vacuum strength was observed, (approx. 10\% per $13 \mathrm{kPa}$ ), Fig. 13. The same conclusion about the linear proportionality between strain and vacuum strength, as drawn from the kymograph, is again confirmed. At the maximum vacuum strength of $98.7 \mathrm{kPa}$, the strain of the membrane reaches $69 \%$. This performance meets the requirement of most current cell stretching experiments. Our device outperforms the highly popular commercial device, the Flexcercell unit, as well as most of other microscale devices reported in the recent literature.

\subsection{Cell stretching experiment}

Preliminary cell stretching experiments were carried out to demonstrate the capability of the device for applying a strain on cells. Two different cell lines, monkey fibroblast (COS-7) and benign human foreskin fibroblast (Fibro) were separately used in two stretching experiment. Standard protocol for PDMS substrate cell culture was used for coating the membrane with collagen gel. 
The cells suspended in the culture medium at a number concentration of 1 million $/ \mathrm{ml}$ were loaded by a pipette. The relatively low concentration ensured the isolation of cells, such that their boundaries are easily traceable. The pipette tip loaded with $10 \mu \mathrm{l}$ solution was then inserted into the channel inlet, while another empty pipette tip was inserted in the channel outlet to gently redraw a volume of $5 \mu \mathrm{l}$. Subsequent incubation $\left(5 \% \mathrm{CO}_{2}, 37^{\circ} \mathrm{C}\right)$ for 1 to 3 hours allows the cells to form attachment with the PDMS membrane. Fresh medium was added into the channel from one end. Driven by gravity, the fresh medium slowly flowed across the channel and replaced the consumed medium. The cells were further incubated until they fully spread on the membrane. The chip was then transferred onto the microscope stage and connected to the pneumatic control system for stretching experiments.

In the stretching experiment, strains higher than $12 \%$ were loaded on each cell while image recordings were taken in real-time with differential interference contrast (DIC) microscopy under $20 \times$ magnification. Two snapshots were extracted from each recording at the relaxed and stretched states of the membrane respectively. The cell outline in each snapshot was traced by ImageJ. The enlargement of cell projected area is clearly observed from the comparison of their traced outline. Therefore, it proved that the cells have maintained firm attachment with the membrane, and hence the strain on the membrane could be successfully transferred to the cell. The device was tested with a stretching frequency of $0.1 \mathrm{~Hz}$ and a strain of $10 \%$ over 4 hours. No leakage and change in performance were observed.

\section{Conclusion}

We designed, fabricated and tested a side-stretching device. A 2D numerical model was analyzed using finite element method. The simulation predicts the strain uniformity, strain-vacuum linear proportionality and provides guidelines for sizing the geometry parameters of the device. A device with ultra-thin bottom and a $3 \times 5$ array of stretching units was successfully fabricated using standard fabrication techniques of microfluidics. A novel alignment and bonding system developed in-house to assist the accurate and fast bonding process of two PDMS parts with microstructures. A programmable control system was also developed to provide individual pneumatic actuations to each unit of the device. Additional modifications to the control system was made to allow time lapse recordings for long-term cell stretching experiments. The upper limit of the stretching frequency was determined by the performance of the vacuum source. Mechanical characterization of the device was performed both qualitatively and quantitatively. The predictions from the numerical simulation were confirmed while the in-focus stretching motion was also verified. Finally, two types of cells were cultured and stretched in the device. The effective transferral of strain from the device membrane to the cells was demonstrated. 
Acknowledgements The authors would like to acknowledge the generous funding from Mechanobiology Institute, Singapore. We thank Michael Sheetz for initiating this project. We are also grateful to the advices from Leyla Kocgozlu, Benoit Ladoux on the device modification, assistance from Lok Khoi Seng, Lee Peng Foo for the biological experiments and support from Su Maohan as well as Wu Min for microscopic imaging.

\section{References}

1. T.D. Brown et al., Am. J. Med. Sci. 316, 162-168 (1998)

2. M. J. Davis, J. A. Donovitz, and J. D. Hood, Am. J. Physiol. 262, C1083C1088 (1992)

3. D. Huh et al., Science 328, 1662-1668 (2010)

4. D. E. Ingber, Ann. Med. 35, 564-577 (2003)

5. Flexcell International Corporation, FX-4000 Flexcell Tension Plus and Tissue Train System Specifications (2007)

6. J. Garvin et al., Tissue Eng. 9967979 (2003)

7. L. Huang, P. S. Mathieu, and B. P. Helmke, Ann. Biomed. Eng. 3817281740 (2010)

8. Y. Huang et al. Nanomedicine 8, 543-553 (2013)

9. Y. Kamotani et al., Biomaterials 29, 2646-2655 (2008)

10. N. Lindqvist et al., Invest. Ophthalmol. Vis. Sci. 51, 16831690 (2010)

11. T. Mawatari et al., J. Orthop. Res. 28, 907-913 (2010)

12. C.F. Lee et al.,Biochem. Biophys. Res. Commun. 401344349 (2010)

13. A.J. Maniotis, C. S. Chen, and D. E. Ingber, Proc. Natl. Acad. Sci. USA 94, 849854 (1997)

14. T. Mizutani, H. Haga and K. Kawabata, Acta Biomater. 3, 485493 (2007)

15. B.N. Nguyen, J. Chetta and S.B. Shah, Cell. Mol. Bioeng., 5 504-513 (2012)

16. C. Moraes et al., Lab Chip 10, 227-234 (2010)

17. C. Moraes, Y. Sun and C.A. Simmons, Integr. Biol. 3, 959-971 (2011)

18. I. Owan et al. Am., J. Physiol. - Cell Physiol. 273, C810-C815 (1997)

19. B.J. Pfister et al., J. Neurosci. 24, 79787983 (2004)

20. C.S. Simmons et al., J. Micromech. Microeng. 21, 227-234 (2010)

21. N.J. Sniadecki et al., Rev. Sci. Inst. 79044302 (2008)

22. Y. Shi, et al., J. Cell. Physiol. 226, 21592169 (2011)

23. W. Tan et al., Biomed. Microdevices 10, 869-882 (2008)

24. E. G. Vanderploeg, C.G. Wilson and M.E., Osteoarthr. Cartilage 16, 1228-1236 (2008)

25. K. Shimizu et al., Sensor. Actuat. B-Chem. , 156, 486-493 (2011)

26. E. Shojaei-Baghini, Y. Zheng and Y. Sun, Ann. Biomed. Eng. 41, 1208-1216 (2013)

27. D. Wang et al. Integr. Biol. (Camb)2 288-293 (2010)

28. M.H. Wu et al. Biomed. Microdevices 11, 1-10 (2011) 

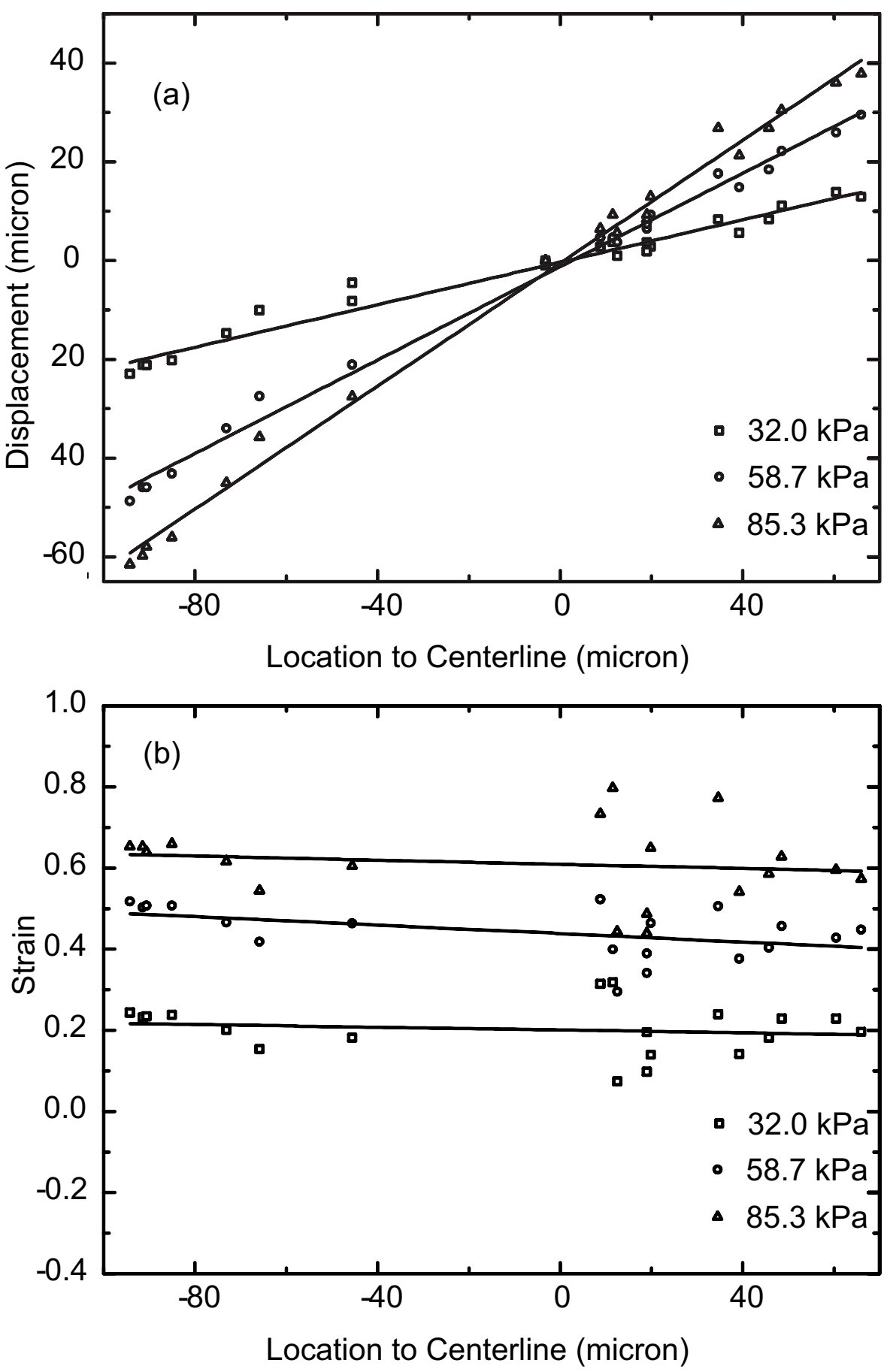

Fig. 13 Measured results of the stretching experiment: (a) Displacement versus location at different vacuum strengths; (b)Strain versus location at different vacuum strengths. 


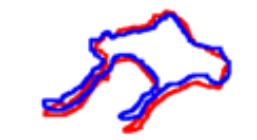

(a)

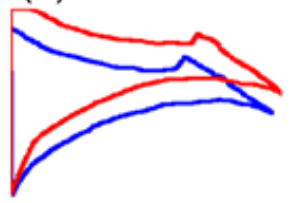

(b)

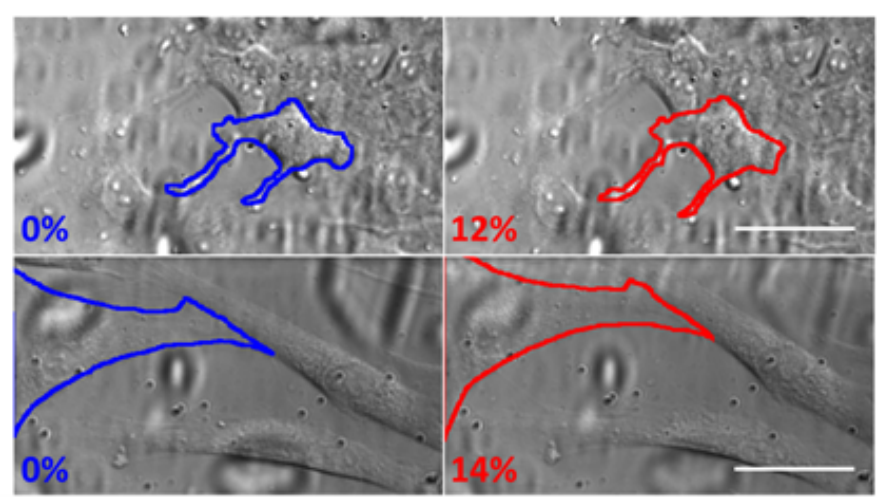

Fig. 14 Comparison of cell outline at stretched and relaxed states of (a) COS-7 and (b) Fibro. The labeled strain is strain on the membrane measured by the displacement of beads. Reference bar is $50 \mu \mathrm{m}$. 